\title{
Regulation of Astrocyte Proliferation by FGF-2 and Heparan Sulfate in vivo
}

\author{
Fernando Gómez-Pinilla, Lawrence Vu, and Carl W. Cotman \\ Department of Neurology and IRU in Brain Aging, University of California, Irvine, California 92717
}

The goal of this study was to examine the ability of basic fibroblast growth factor (FGF-2) to promote reactivity and/or proliferation of astrocytes in vivo following brain injury, and the possible mechanisms involved. A small bilateral lesion in the motor-sensory cortex was performed, and either FGF-2, FGF-2 plus heparan sulfate, heparan sulfate, or saline was applied unilaterally in a piece of Gelfoam within the wound cavity. Following lesions, there was an increase in FGF-2 and FGF receptor (FGFR) immunoreactivities in the area surrounding the lesion in all the treatment groups. Rats that received treatment with recombinant FGF-2 alone showed an increase in the density of astrocytes as compared to the control group. The same group of rats exhibited an increase in the density of cells displaying FGF-2 immunoreactivity and cells displaying FGFR-1 immunoreactivity, and also an induction of FGF-2 mRNA in the tissue surrounding the lesion. The group of rats that received FGF-2 combined with heparan sulfate showed a larger increase in the same cellular parameters. Our results suggest that the FGF-2/FGFR system is involved in the regulation of astrocytic reactivity and/or proliferation in the brain and its action is potentiated by heparan sulfate. The action of FGF-2 on CNS injury appears to be part of an autocrine cascade that involves induction of FGF-2 and its receptor, thereby enhancing the ability of astrocytes to respond to FGF-2.

[Key words: neuronal death, plasticity, trauma, immunohistochemistry, mRNA, FGF receptor, growth factors]

Injury to the CNS triggers a sequence of cellular events in the remaining tissue, including death of neurons and profuse proliferation of astroglia. Primed by the original insult, a cascade of biochemical events takes place at the site of the injury, triggering the release of diffusible molecules that appear to regulate glial proliferation, neuronal survival, or regrowth. The response of astrocytes to injury is central to the events leading to the survival and healing of the tissue affected by the insult. Proliferation of astrocytes around the injury site contributes to the restoration of the blood-brain barrier and provision of trophic factors to the damaged tissue. Paradoxically, the same astrocytes form a bar-

\footnotetext{
Received July 18, 1994; revised Aug. 30, 1994; accepted Sept. 7, 1994.

This work was supported by AG00538 and the American Paralysis Association. We thank Dr. David Cribbs for performing the dot blot experiments, James Choi for his help in the analysis of the data, and Dr. Jennifer Kahle for critical review of the manuscript.

Correspondence should be addressed to Fernando Gomez-Pinilla, Ph.D., Department of Neurology/IRU in Brain Aging, Biological Science II (2205), University of California, Irvine, CA 92717-4550.

Copyright (C) 1995 Society for Neuroscience 0270-6474/95/152021-09\$05.00/0
}

rier around the injury site that is almost impenetrable to the ingrowing neuritic processes. Accordingly, the elucidation of the mechanisms that control the differentiation, reactivity, and/or proliferation of astrocytes is important in the development of therapeutic procedures to enhance neuronal survival and to foster regeneration following brain trauma.

Substances that control astrocytic reactivity and/or proliferation include trophic molecules that are released by cells surrounding the injury. Fibroblast growth factors (FGFs) are typically recognized for their wide spectrum of action exerted on a large variety of cell types including astrocytes and neurons. In the brain, the best characterized member of the FGF family is basic FGF (bFGF or FGF-2), which is active as a promoter of neuronal survival and neurite extension in vitro (Walicke, 1988a,b; Grothe et al., 1989) and in vivo (Sievers et al., 1987; Anderson et al., 1988; Otto et al., 1989). FGF-2 is primarily expressed by astrocytes and appears to participate in their differentiation (Lillien and Raff, 1990; McKinnon et al., 1990) and proliferation (Morrison and De Villis, 1981). Therefore, FGF-2 appears to be a good candidate for a role in the regulation of astrocytic function in the healthy, injured, or diseased CNS.

A large body of evidence indicates that heparan sulfate proteoglycans (HSPG) participate in the regulation of FGF-2 action (Ruoslahti and Yamagushi, 1991). Indeed, HSPG acts as a lowaffinity receptor for FGF-2 (Moscatelli, 1987; Klagsbrun and Baird, 1991), potentiating the action of FGF-2 and protecting it from degradation. Recent studies indicate that the binding of FGF-2 to high-affinity receptors requires the presence of heparin or cell-derived HSPG (Rapraeger et al., 1991; Yayon et al., 1991). Apparently, heparin interacts independently with a portion of the transmembrane tyrosine kinase glycoprotein component of the FGF receptor complex (Kan et al., 1993). Four types of high-affinity FGF receptors (FGFR) have been identified, FGFR-1 or $f l g$ (Lee et al., 1989), FGFR-2 or bek (Dionne et al., 1990; Mansukhani et al., 1992), FGFR-3 (Keegan et al., 1991), and FGFR-4 (Partanen et al., 1991). FGFR-1 consists of three extracellular immunoglobulin-like domains, a transmembrane domain, and an intraccllular tyrosine kinase domain (Lce et al., 1989; Ullrich and Schlessinger, 1990). FGF-2 does not have a signal peptide controlling its own release from cells, and to a large extent the effect of FGF-2 appears to be regulated by the interaction with its receptor. Increasing evidence indicates that FGFR-1 is induced by brain trauma (Gómez-Pinilla and Cotman, 1992; Logan et al., 1992) and afferent activity (Van der Wal et al., 1994), suggesting that the FGFR-1 may have a role in brain plasticity.

In the present study we present evidence supporting an action of FGF-2 on astrocytic reactivity and/or proliferation in vivo. 
Furthermore, we examine the possibility that FGF-2 on astrocytic reactivity and/or proliferation may be modulated by an interaction with extracellular matrix components. According to this hypothesis interactions between FGF-2, FGFR-1, and HSPG may regulate the action of FGF-2 in brain injury.

\section{Material and Methods}

Surgery and experimental procedures. Sprague-Dawley male rats 3-4 months old received aspiration of a small segment of the motor-sensory cortex $(1.5 \mathrm{~mm}$ diameter, $1.7 \mathrm{~mm}$ deep from top of skull, $3.5 \mathrm{~mm}$ lateral to the midline) in both hemispheres. Groups of rats $(n=5)$ received a piece of Gelfoam embedded with human recombinant FGF-2 (100 $\mathrm{ng} / \mathrm{ml}$; endotoxin < $3.0 \mathrm{Ue} / \mathrm{mg} ; \mathrm{UBI}, \mathrm{NY}$ ) dissolved in PBS, heparan sulfate (HS; $10 \mu \mathrm{g} / \mathrm{mnl}$; sodium salt, bovine kidney; Sigma) dissolved in PBS, or both, in the right hemisphere. The wound cavity of the left hemisphere received a piece of Gelfoam embedded with saline. Group of rats $(n=4)$ to be used in nuclease protection assay received FGF2, HS, or FGF-2/HS treatments bilaterally. Rats to be used as a control $(n=5)$ received a piece of Gelfoam embedded with saline bilaterally in the cerebral cortex. These experiments were performed according to NHI guidelines for the care of animals and studies have been approved by the animal care committee.

Two days after surgery, rats to be used for immunohistochemical experiments were deeply anesthesized with Nembutal $(75 \mathrm{mg} / \mathrm{kg}$, i.p.) and perfused with $200 \mathrm{ml}$ of phosphate-buffered saline followed by 400 $\mathrm{ml}$ of $4 \%$ paraformaldehyde in $0.1 \mathrm{M}$ Sorensen buffer; the brains were removed, postfixed for $15 \mathrm{~min}$ in fresh solution of the same fixative, and stored overnight in $20 \%$ sucrose. Serial coronal sections $(30 \mu \mathrm{m})$ throughout the forebrain were cut in a cryostat and processed for FGF2, FGFR-1, or glial fibrillary acidic protein (GFAP) immunoreactivities. Alternate sections were stained with cresyl violet to visualize the general cytoarchitecture of the areas under study. Rats to be used in nuclease protection assays were quickly decapitated, and an area $(0.5 \mathrm{~cm})$ of cerebral cortex around the wound cavity was dissected out and frozen in dry ice.

Immunohistochemistry. The tissue sections were first treated with $1 \%$ $\mathrm{H}_{2} \mathrm{O}_{2}$ in cold $100 \%$ methanol to inactivate the endogenous peroxidase activity. Alternate sections were then incubated overnight in one of three antibodies: (1) a mouse monoclonal anti-FGF-2 $(4 \mu \mathrm{g} / \mathrm{ml}$; UBI, NY), which recognizes biologically active FGF-2 and does not crossreact with FGF-1 (Matsuzaki et al., 1989); (2) a mouse monoclonal (2 $\mu \mathrm{g} / \mathrm{ml}$; UBI, NY) that specifically recognizes the external domain of the FGF receptor [ $f g$ or FGFR-1; Western immunoblot analysis determined that this antibody was able to detect $50-100$ ng of human FGF receptor protein of the flg type (Hou et al., 1991)]; (3) a rabbit monoclonal anti-GFAP $(2.9 \mu \mathrm{g} / \mathrm{ml}$; Dakopatts Labs, Denmark), diluted in PBS containing 0.1 Triton X-100 and 2\% BSA. The sections were then rinsed three times over $30 \mathrm{~min}$ and incubated in rat-adsorbed biotinylated horse anti-mouse immunoglobulin G (FGF-2 and FGFR-1 immunostaining) or biotinylated goat anti-rabbit IgG (GFAP staining) for $1 \mathrm{hr}$. After several rinses, sections were incubated in avidin-HRP complex for $1 \mathrm{hr}$, rinsed, incubated in 3,3'-diaminobenzidine (DAB; 0.5 $\mathrm{mg} / \mathrm{ml}$ in Tris buffer, $10 \mathrm{~min}$ ), and then reacted for $5 \mathrm{~min}$ with fresh DAB solution containing $0.01 \% \mathrm{H}_{2} \mathrm{O}_{2}$. All the sections were thoroughly rinsed with PBS, mounted, dehydrated with increasing gradients of ethanols, and coverslipped with Depex mounting medium. Sections were examined on an Olympus BH-2 microscope. Immunohistochemistry controls included (1) incubation in buffer excluding the primary antibody, (2) incubation with a secondary antibody against a wrong species, and (3) incubation with a preabsorbed anti-FGF-2 antisera [10 $\mu \mathrm{g}$ of recombinant FGF-2 (UBI, NY)/ml of normally diluted serum; $15 \mathrm{hr}$ ]. The results of these immunohistochemistry controls were consistently negative. FGF-2 staining was abolished in cell structures but was slightly increased in the extracellular matrix and blood vessels.

To further characterize the anti-FGF-2 antibody, dot blot experiments were performed (according to UBI protocols) to investigate if this an tibody also binds FGF-2 complexed to heparan sulfate. Briefly, recombinant FGF-2 $(6.25 \mathrm{pg} / \mathrm{ml}$ to $0.1 \mu \mathrm{g} / \mathrm{ml}$; UBI) was combined with HS $(0,10,100 \mu \mathrm{g} / \mathrm{ml}$; Sigma $)$, and then the anti-FGF-2 antibody was added at a final concentration of $4 \mu \mathrm{g} / \mathrm{ml}$. The signal related to the staining with the anti-FGF-2 antibody was not affected by the presence of HS The results of these experiments showed that the binding of the antiFGF-2 antibody to FGF-2 is independent of the presence of heparan sulfate.
Double-staining immunohistochemistry. To ascertain the cellular localization of FGF-2 or FGFR-1, sections were costained with a second antibody. An anti-GFAP antibody was used to detect astrocytes. The color reaction to detect the first antibody (anti-FGF-2) was developed with nickel chloride combined with DAB, giving a purple reaction product instead of the typical brown reaction product. Sections were thoroughly rinsed in PBS, reacted for $15 \mathrm{~min}$ in excess avidin, and then rinsed for another $15 \mathrm{~min}$ in PBS with excess biotin. After rinsing in PBS, sections were incubated overnight in anti-GFAP antibody. The rest of the process is the same as described above, except that the second antibody was detected with $\mathrm{DAB}$ alone, resulting in a brown reaction product.

Quantification procedure. To assess the effects of the lesion and treatments, cells immunostained for GFAP, FGF-2, or FGFR-1 contained within a $0.25 \mathrm{~mm}^{2}$ area positioned at $240 \mu \mathrm{m}$ from the edge of the lesion were counted bilaterally. Cells were counted under camera lucida microscopy at $160 \times$ magnification at four coronal planes separated by about $100 \mu \mathrm{m}$ from each other. To minimize variability between animals, the ratio between the ipsilateral and contralateral sides to the treatment was calculated for each rat. The ratio mean values for GFAP, FGF-2, or FGFR-1 were compared across the different experimental conditions using ANOVA and Fisher's test, and the data are expressed as a percentage of controls.

Nuclease protection assay. Total cellular RNA was isolated by guanidine thiocyanate extraction according to Chomczynski and Sacchi (1986). Quantification of total RNA was performed by absorption at $260 \mathrm{~nm}$. Briefly, total RNA $(30 \mu \mathrm{g})$ from particular brain regions was dissolved in $30 \mu \mathrm{l}$ of hybridization buffer $(80 \%$ formamide, $40 \mathrm{mM}$ PIPES pH 6.4, $400 \mathrm{~mm}$ sodium chloride, and $1 \mathrm{~mm}$ EDTA) containing $5 \mu$ of a ${ }^{32} \mathrm{P}$-labeled FGF-2 cRNA probe (specific activity, $10^{\circ} \mathrm{cpm} / \mu \mathrm{g}$ of RNA). After being heated at $85^{\circ} \mathrm{C}$ for $10 \mathrm{~min}$ to denature RNA, the cRNA probe was allowed to anneal the endogenous RNA at $55^{\circ} \mathrm{C}$ overnight. At the end of the hybridization, the solution was diluted with RNase digestion buffer containing $40 \mu \mathrm{g} / \mathrm{ml}$ of RNase A and $2 \mu \mathrm{g} / \mathrm{ml}$ of RNase $T_{1}$ and incubated for $30 \mathrm{~min}$ at $37^{\circ} \mathrm{C}$. Following proteinase K $(240 \mu \mathrm{g} / \mathrm{ml})$ digestion, samples were extracted with phenol/chloroform and ethanol precipitated. For the pellet containing the RNA, RNA hybrid was resuspended in gel loading buffer, boiled at $85^{\circ} \mathrm{C}$, and separated on a $5 \%$ polyacrylamide, $7 \mathrm{~m}$ urea gel unit. The gel was dried and the protected fragment was visualized by autoradiography on Bmax-hyperfilm (Amersham, IL). In some experiments the same total RNA used for FGF-2 mRNA measurements was hybridized with a rat glyceraldehyde 3-phosphate dehydrogenase cRNA probe (GAPDH; Ambion, TX) to ensure that samples used for the assay contained an equivalent amount of RNA. FGF- 2 mRNA contents were estimated by measuring the peak densitometry area of the autoradiogram analyzed with an image analysis system (Imaging Inc., St. Catherines, Ontario, Canada). The ratio FGF-2:GAPDH of optical densitometric values was calculated for each treatment and is expressed as relative to control.

To assess relative levels of FGF-2 mRNA expression, we used an FGF-2 32 -labeled cRNA probe prepared from cDNA templates coding the rat FGF-2 gene (kindly provided by Dr. Andrew Baird; Shimasaki et al., 1988). A pBluescript SK+ plasmid (RObFGF503) was linearized with $\mathrm{NcoI}$ and then transcribed using T7 RNA polymerase to generate a 524 base antisense probe that contained a 477 base FGF-2 gene coding region. To be used as a control, a 376 base GAPDH cRNA probe was generated by transcribing with T3 RNA polymerase, a manufacturerprelinearized plasmid (Ambion, TX). Transcription reactions were performed using a Promega transcription kit (Promega, WI) and $\alpha-{ }^{32} \mathrm{P}-\mathrm{CTP}$ (Amersham, IL; $800 \mathrm{Ci} / \mathrm{mmol}$ ).

\section{Results}

Distribution of FGF-2 and FGFR-1 in normal rat brain. We used an affinity-purified monoclonal antibody against the active form of FGF-2 to characterize the cellular localization of FGF-2 in the brain. Double-staining immunohistochemistry using an anti-GFAP antibody and an anti-FGF-2 antibody showed that FGF-2 immunoreactivity was present primarily in astrocytes throughout the brain (data not shown), as previously described in detail elsewhere (Gómez-Pinilla et al., 1992). Within single astrocytes FGF-2 staining was compartmentalized in the nuclear region. To stain cells displaying FGFR-1 immunoreactivity, we 

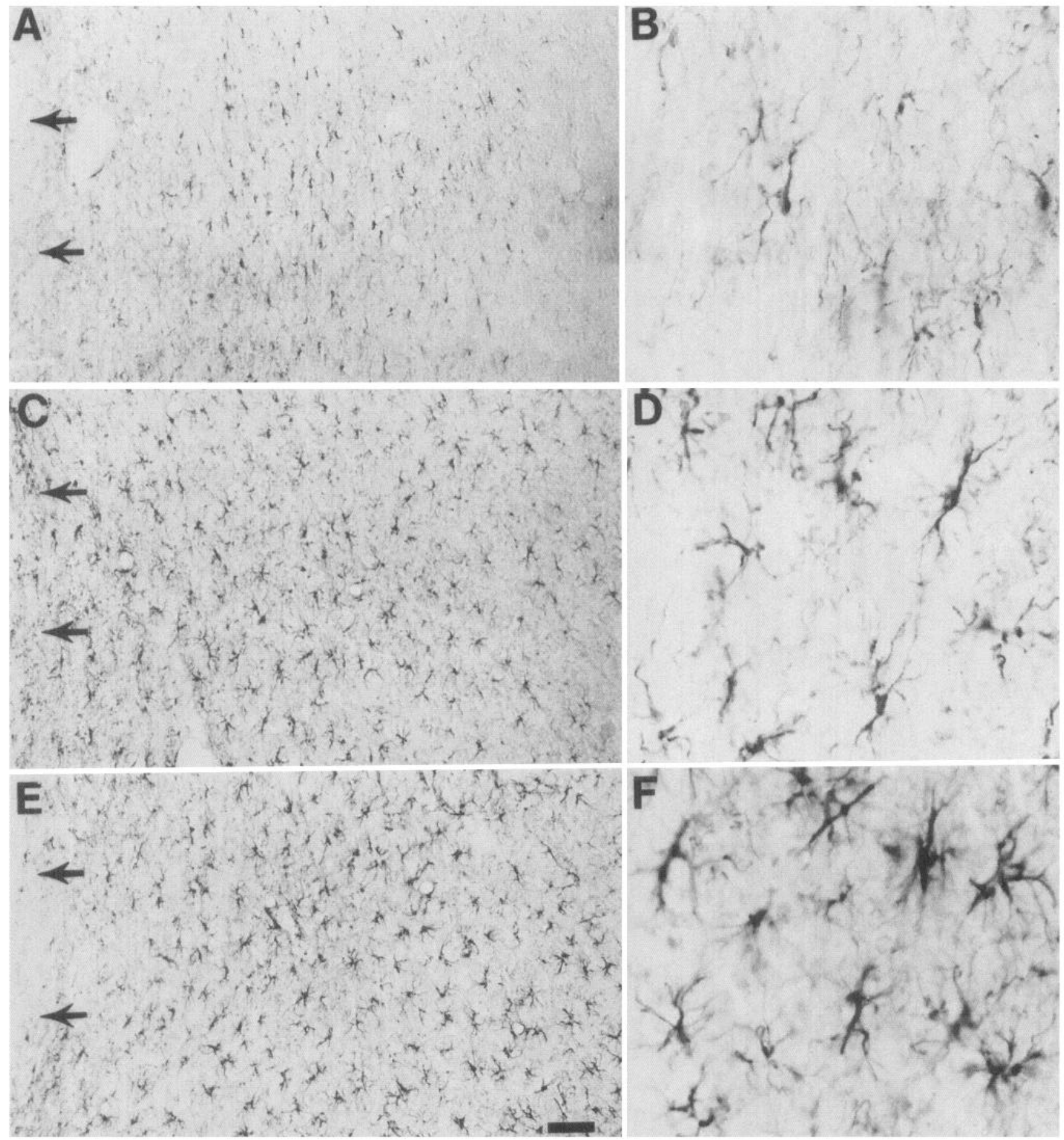

Figure 1. GFAP immunostaining showing the effects of FGF-2 and FGF-2/HS treatments on astrocytes. The photographs were taken from the area adjacent to the wound cavity (double arrows), which was filled with a piece of Gelfoam embedded with the experimental solution. $A$, Sample section from a saline control animal showing the general distribution of astrocytes by the site of injury (arrows). $B$, High magnification of $A$ displaying the typical morphology of astrocytes by the injury site. $C$, Effects of recombinant FGF-2 treatment on the distribution and morphology of astrocytes. FGF-2 applied within the wound cavity caused an increase in number and intensity of GFAP staining in astrocytes. D, High magnification of $C$ showing the effects of FGF-2 treatment on the morphology of astrocytes. There was an increase in astrocytic density and intensity of GFAP staining compared with saline or HS treatments (not shown). E, Effects of the combined application of HS and FGF-2. There was an increase in the density number of astrocytes and intensity of GFAP staining compared either with saline, HS, or FGF-2 treatments. $F$, High magnification of $E$ showing the morphology of astrocytes. Scale bar (in $E$ ), $60 \mu \mathrm{m}$ for $A, C$, and $E ; 15 \mu \mathrm{m}$ for $B, D$, and $F$.

used a monoclonal antibody specific against FGFR-1. FGFR-1 immunoreactivity was light and sparse in the intact brain but occasionally present in isolated cells mostly within the cerebral cortex and hippocampal formation. Double-staining immunoh- istochemistry showed that FGFR-1 immunoreactivity (data not shown) was colocalized with GFAP immunoreactivity revealing that FGFR-1 was primarily expressed in astrocytes, as previously demonstrated (Van der Wal et al., 1994). 


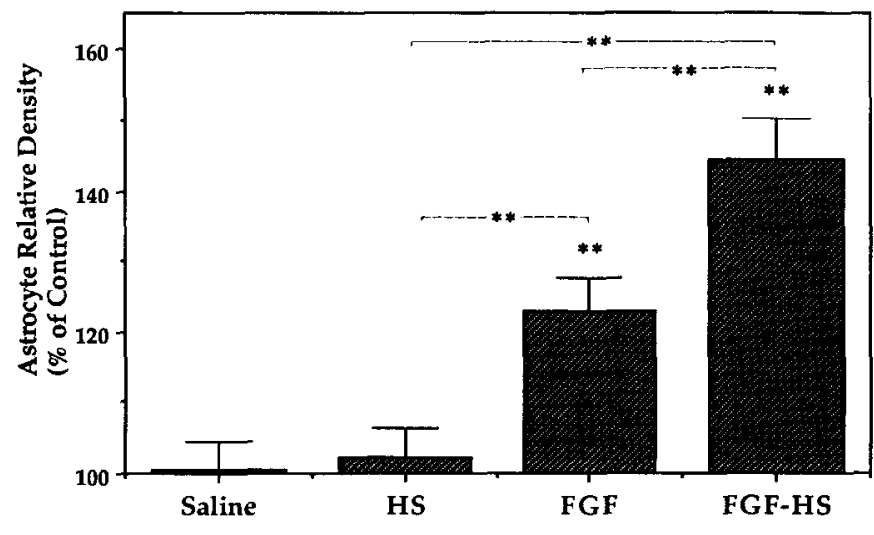

Figure 2. Quantification of the number of astrocytes immunostained with an anti-GFAP antibody near the area surrounding the wound cavity. The graph shows the effects of HS, FGF-2, and FGF/HS on the density of astrocytes, expressed as a percentage of control (saline). FGF-2 treatment elicited an increase in the number of astrocytes as compared with saline treatment, and this effect was potentiated by the combined application of FGF/HS. Statistical comparisons were performed using ANOVA and Fisher's test $\left({ }^{* *}, P<0.01\right)$. Error bars are SD.

Induction of FGF-2 and FGFR-1 after lesion. An anti-GFAP antibody was used to evaluate changes in the astrocyte population following injury. Two days after performing a cortical lesion there was a large increase in the density of astrocytes in the area surrounding the injury site (Figs. 1A, 2). Parallel sections stained with an anti-FCF-2 antibody showed a large increase of astrocyte-like cells displaying FGF-2 immunoreactivity near the lesion area as previously demonstrated (Gómez-Pinilla and Cotman, 1992; Gómez-Pinilla et al., 1992; Figs. 3A, 4). These FGF-2-positive astrocytes responding to the lesion stimulus showed signs of hypertrophy such as FGF-2 staining in the cytoplasmic region and proximal processes.

Two days after cortical lesion, the arca surrounding the lesion also showed a large increase in the density of cells displaying FGFR-1 immunoreactivity (Figs. $5 A, 6$ ). The majority of these FGFR-1 cells were astrocytes as revealed by costaining with an anti-GFAP antibody (data not shown).

Effects of exogenous FGF-2 on astrocytes, FGF-2 cells, and FGFR-1 cells. To examine a possible effect of FGF-2 on astrocytic reactivity and/or proliferation, we increased the availability of FGF-2 to the cells located near the injury site by placing FGF-2-embedded Gelfoam within the lesion cavity. Two days following the priming lesion in the cerebral cortex and FGF-2 treatment, there was an increase in the density of astrocytes immunostained with the anti-GFAP antibody as compared to control rats that received saline only $(P<0.01$; Fig. 2$)$. This FGF2 -induced increase of astrocytes was particularly obvious along the periphery of the cortical lesion where the FGF-2-embedded Gelfoam was applied. These astrocytes appeared to stain stronger for GFAP (Fig. IC,D) than saline-treated astrocytes (Fig. $1 A, B)$.

Analysis of the tissue sections stained for FGF-2 in parallel with the GFAP staining showed that the recombinant FGF-2 applied in the wound cavity also had an effect on the population of FGF-2-stained astrocytes. These astrocytes showed FGF-2 staining along their processes (Fig. $3 C, D$ ) in contrast to the typical FGF-2 staining only in the nuclear region (Fig. $3 A, B$ ). The number of cells displaying FGF-2 staining by the lesion area was increased in the FGF-2-treated group compared to control rats treated with saline ( $P<0.01$; Fig. 4).

Another set of tissue sections was stained in parallel with an anti-FGFR-1 antibody. Microscopic examination of these sections revealed that there was an induction of cells displaying FGFR-1 immunoreactivity. These cells resembled astrocytes and developed a stronger FGFR-1 staining in the cell body and processes (Fig. 5C,D) as compared to saline-treated cells (Fig. $5 A, B)$. The density of FGFR-1-positive cells in the FGF-2-treated group was significantly increased relative to control rats treated with saline $(P<0.01$; Fig. 6).

A separate group of rats $(n=4)$ was treated bilaterally with recombinant FGF-2 and the tissue surrounding the wound cavity removed. This tissue was assayed via nuclease protection assay to examine the relative levels of FGF- 2 mRNA. Results showed an increase in FGF-2 mRNA in the group of rats treated with FGF-2 relative to the saline control rats (optical density ratio FGF-2:GAPDH $=1.25, n=4$; Fig. 7).

Effects of exogenous $H S$ on astrocytes, FGF-2 cells, and $F G F R-1$ cells. Several lines of evidence indicate that HS has an important role in the physiology of FGF-2. To test the effect of HS alone in our lesion paradigm, a piece of Gelfoam embedded with HS was applied unilaterally into the wound cavity. Quantification of the number of GFAP-stained astrocytes did not show any significant difference between the HS-treated group versus the saline-treated group of rats (Fig. 2). Similarly, quantification of FGF-2-stained cells did not show a significant difference between HS-treated rats versus saline-treated control rats (Fig. 4). However, analysis of the FGFR-1-immunostained cells showed a significant increase in density in the rats treated with $\mathrm{HS}$ as compared to the control rats treated with saline $(P<0.01$; Fig. 6).

A group of rats was treated bilaterally with $\mathrm{HS}$ and the tissue surrounding the wound cavity dissected out. The tissue was analyzed using a nuclease protection assay to examine the relative levels of FGF-2 mRNA. Results showed no apparent changes in levels of FGF-2 mRNA in the HS-treated rats relative to the saline control rats (optical density ratio FGF-2:GAPDH $=0.92$, $n=4$; Fig. 7).

Effects of exogenous FGF-2 combined with HS on astrocytes, FGF-2 cells, and FGFR-1 cells. Next, we examined the possibility that the combination FGF-2/HS could potentiate the action of FGF-2 alone. Accordingly FGF-2/HS was applied in a piece of Gelfoam placed into the wound cavity. The FGF-2/HS application triggered a larger increase of the same cellular parameters that had been increased by separate applications of either FGF-2 or HS. Specifically, rats receiving FGF-2/HS treatment displayed a significant increase in the number of astrocytes relative to control rats treated with saline $(P<0.01$; Fig. 2$)$, or rats receiving HS treatment $(P<0.01$; Fig. 2$)$. FGF-2-immunostained cells in the FGF-2/HS group were significantly increased in number relative to the control rats treated with saline $(P<0.01$; Fig. 4$)$ or the rats receiving HS treatment $(P<0.01$; Fig. 4$)$ or FGF-2 treatment $(P<0.01$; Fig. 4). FGFR-1-immunostained cells from the FGF-2/HS group also were significantly increased in number relative to the rats receiving $\mathrm{HS}$ treatment $(P<0.01$; Fig. 6$)$ or control rats treated with saline $(P<0.01$; Fig. 6). Immunostaining for GFAP, FGF-2, and FGFR-1 appeared much stronger in the cell bodies and processes of FGF-2/HS-treated rats as compared to saline- or HS-treated cells.

The tissue surrounding the wound cavity from a separate group of rats was treated with $\mathrm{HGF}-2 / \mathrm{HS}$. A nuclease protection 

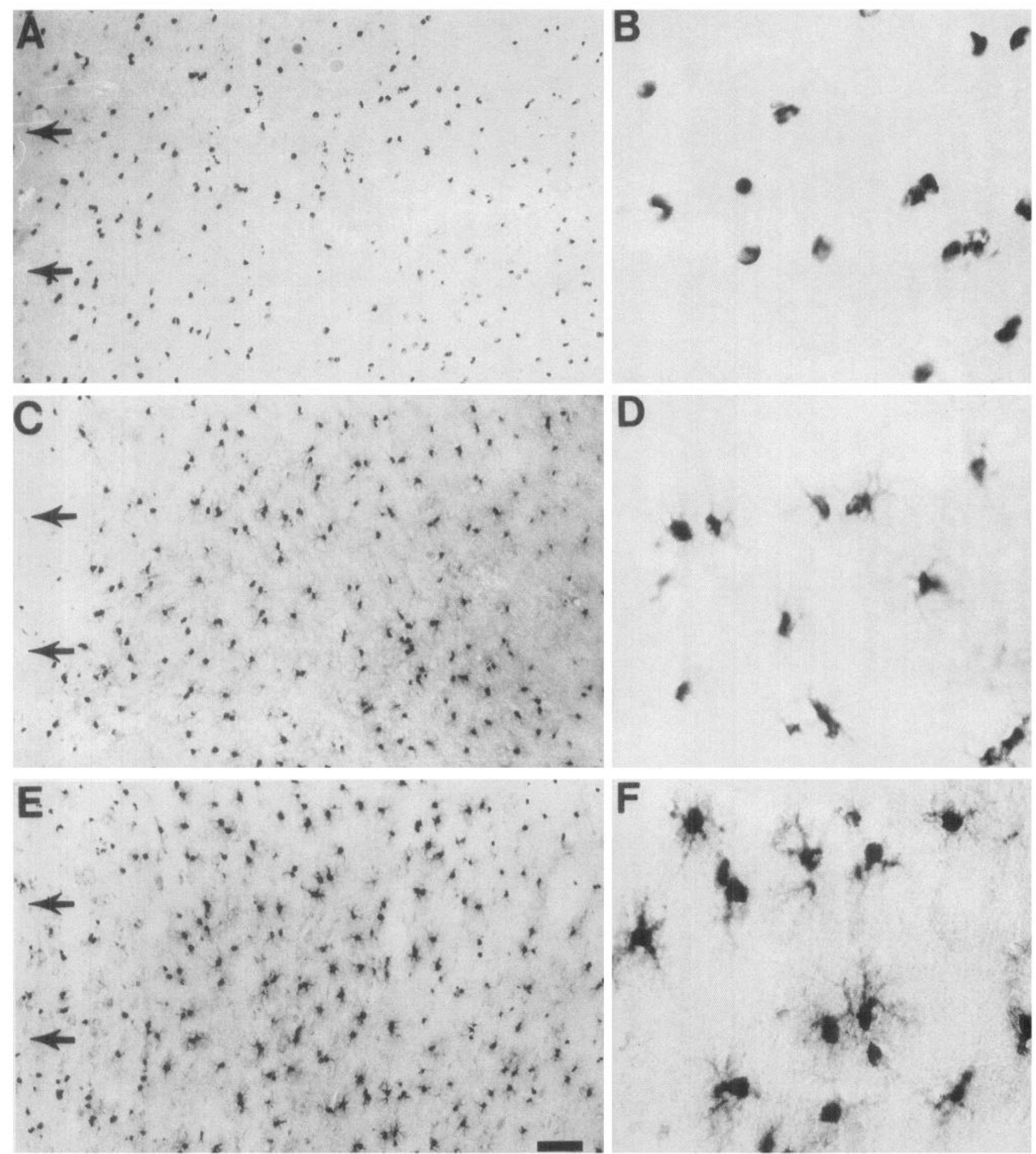

Figure 3. Immunostaining showing the effects of FGF-2 and FGF-2/HS treatments on cells expressing FGF-2 phenotype. The photographs were taken from the area adjacent to the wound cavity (arrows at the edge), which was filled with a piece of Gelfoam embedded with the experimental solution. A, Sample section from a control animal showing the distribution of FGF-2 staining by the periphery of the injury site (arrows). FGF-2 staining was mostly located in astrocytes. $B$, High magnification of $A$ displaying the typical distribution of FGF-2 staining in astrocytes by the injury site. $C$, Effects of recombinant FGF-2 on the distribution and morphology of FGF-2-immunostained cells. FGF-2 treatment caused an increase in number and intensity of FGF-2 staining in astrocytes. $D$, High magnification of $C$ showing the effects of FGF-2 treatment on the phenotypic expression of FGF-2. There was an increase in density of FGF-2 cells and intensity of staining in astrocytic processes compared to treatments with saline or HS. $E$, Effect of the combined application of HS and FGF-2. There was an increase in the density number of FGF-2-positive cells and intensity of FGF-2 staining in processes as compared either with saline, HS (not shown), or FGF-2 treatments. $F$, High magnification of $E$ showing the morphology of FGF-2-stained astrocytes. Scale bar (in $E$ ), $60 \mu \mathrm{m}$ for $A, C$, and $E ; 15 \mu \mathrm{m}$ for $B, D$, and $F$. 


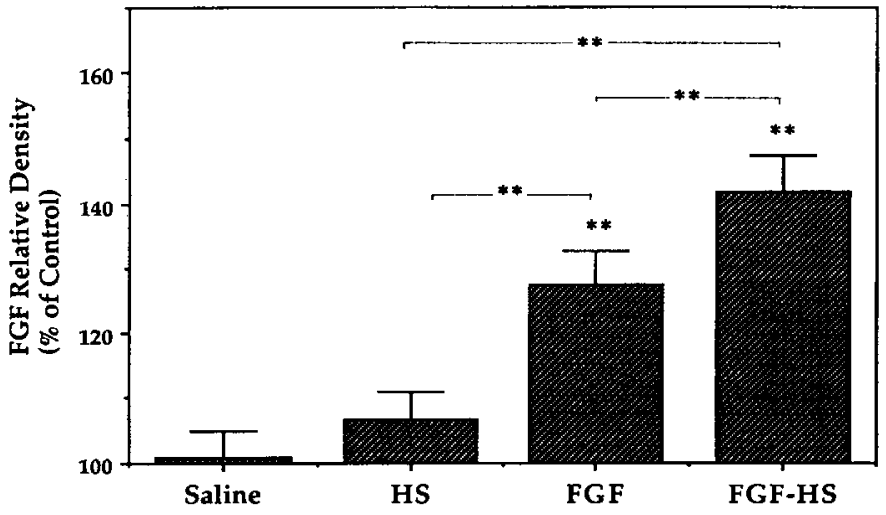

Figure 4. Quantification of the number of FGF-2-immunostained cells by the area surrounding the wound cavity. The graph shows the effects of HS, FGF-2, and FGF/HS on the density of FGF-2-positive astrocytelike cells, expressed as a percentage of control (saline). FGF-2 applied into the wound cavity elicited an increase in the number of FGF-2 cells as compared with saline or HS treatments, and this effect was potentiated by the combined application of FGF/HS. Statistical comparisons were performed using ANOVA and Fisher's test $(* *, P<0.01)$. Error bars are SD.

assay was used to detect relative levels of FGF-2 mRNA. Results showed a relative increase in FGF-2 mRNA in the group of rats that received FGF-2/HS treatment as compared to FGF2-treated rats or saline control rats (Fig. 7). The optical density ratio FGF-2:GAPDH relative to control for the different treatments was $\mathrm{HS}=0.92, \mathrm{FGF}-2=1.25$, and $\mathrm{FGF}-2 / \mathrm{HS}=1.51$ $(n=4)$.

\section{Discussion}

Our results indicate that FGF-2 appears to have a modulatory role in astrocytic reactivity and/or proliferation following CNS traumatic injury. Application of exogenous FGF-2 in the wound cavity triggered an increase in the population of astrocytes by the lesion area. The same intervention specifically increased the number of astrocytes displaying immunoreactivities for FGF-2 and FGFR-1 and also elicited an induction of FGF-2 mRNA in the tissue surrounding the lesion. The effects of FGF-2 on astrocyte reactivity and/or proliferation, and induction of FGF-2 and FGFR-1 were potentiated when recombinant FGF-2 was applied together with HS in the wound cavity.

The observation that exogenous FGF-2 promoted astrocyte reactivity and/or proliferation supports the idea that FGF-2 may stimulate astrocytic proliferation in the brain as predicted by previous studies in vitro. Morrison and De Villis (1981) have demonstrated that FGF-2 promotes proliferation of astrocytes in culture. In addition, our data demonstrate that FGF-2 induces the expression of its own mRNA, supporting the possibility that the action of FGF-2 in astrocyte proliferation may be via regulation of its own synthesis (Flott-Rahmel et al., 1992). Furthermore, our data show that changes in FGF-2 mRNA are translated into protein since there were concomitant changes in FGF-2 immunoreactivity. Our findings are in general agreement with recent studies in culture. Gerdes et al. (1992) have shown that inhibition of the endogenous FGF- 2 expression with antisense oligonucleotides causes a reduction of cell proliferation. FlottRahmel et al. (1992) have demonstrated that treatment of astrocytes or hippocampal neurons in culture with exogenous FGF-2 triggers an induction of FGF-2 gene expression, supporting an autocrine action of FGF-2.
The induction of FGFR-1 by FGF-2 application suggests that the regulation of FGF receptor may be a factor for the regulation of the action of FGF-2. FGF receptor (Gómez-Pinilla et al., 1992; Van der Wal., 1994) and its mRNA (Wanaka et al., 1990; Logan et al., 1992) are present at low levels in the intact brain; however, they are highly regulated by stimuli such as trauma (Logan et al., 1992; Gómez-Pinilla and Cotman, 1992) or physiological activity (Van der Wal et al., 1994). Moreover, the observation that FGF-2 and its receptor were increased adds further support for an autocrine action of FGF-2 on cells.

Current information indicates that $\mathrm{HS}$ has a role in the regulation of the action of FGF-2 on cells; however, the mechanism involved has remained unknown. 'The modulatory role of HS on FGF-2 function is versatile and appears to be achieved by intcracting with multiple targets along the path lcading to the execution of FGF-2 action on cells. For example, HS normally binds FGF-2, which is present in the extracellular matrix or associated with cells. When FGF-2 forms a complex with HS it is more stable than FGF-2 alone and is protected from proteolytic degradation (Gospadorowicz and Cheng, 1986; Flaumenhaft et al., 1990). Interestingly, our data demonstrate a completely new aspect of the interactions between FGF-2 and HS. That is, HS potentiates the effect that FGF-2 exerts on the induction of FGF2 , its mRNA, and its receptor. It is likely that these actions are associated with the regulation of astrocyte proliferation, since the increase in density of astrocytes elicited by FGF-2 was also potentiated by the combined application of FGF-2 and HS.

Thus, based on our results and others, it appears that the modulatory influence that HS exerts on FGF-2 action is partially achieved by an interaction of HS with FGFR. Studies in vitro (Kan et al., 1993) show that the interaction of HS with a specific sequence in one of the immunoglobulin subunits in the extracellular domain of the FGF receptor is independent of FGF binding. According to these investigators (Kan et al., 1993), the FGF receptor is a ternary complex of a HS, tyrosine kinase transmembrane glycoprotein, and ligand, and that HS binding domain is essential for the binding of FGF. Our results are in general agreement with that study and further demonstrate that HS when combined with FGF-2 upregulates FGFR phenotypic expression.

According to our data, the action of FGF-2 may involve a proper balance between FGF-2 and HSPG. HSPGs as well as other extracellular matrix components exhibit different expression patterns (Herdon and Lander, 1990; Sheppard et al., 1991, Fuxe et al., 1994) and different biological activities (Hondermarck et al., 1992; Lander, 1993; Letourneau et al., 1994) throughout ontogeny. For example, Nurcombe et al. (1993) have recently reported that differences in the type of HS carried by a single core protein can switch the affinity for FGF-1 or FGF-2. The changes in the affinities of HS for FGF-1 versus FGF-2 appear to be coordinated according to their particular temporal expressions, which in turn are reflected in specific functional demands. Therefore, different ratios of FGF-2 and particular types of HSPGs may be generated, which may in turn modulate the action of FGF-2 on cells. The induction of the FGF-2/FGFR system by HS is extremely significant in the context of the regulation of the FGF system. The presence of regulatory mechanisms to precisely control FGF-2 signal transduction is important, since FGF-2 is widely distributed in the brain and is very effective in various cellular processes. Increasing evidence indicates that glycosaminoglycans are not randonly distributed in the brain, and their expressions appear to be regulated by functional demand, in particular during de- 

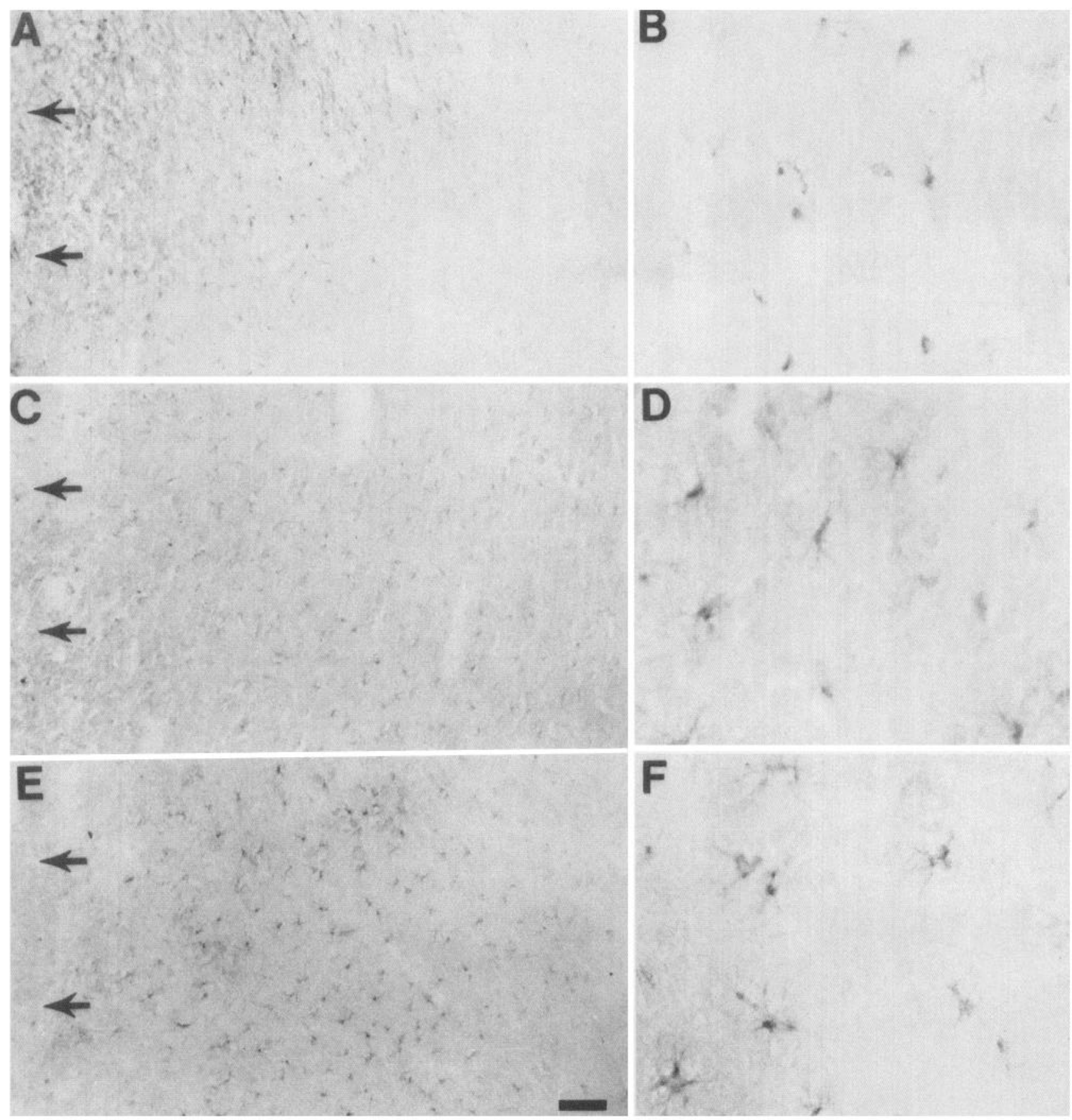

Figure 5. Immunostaining showing the effects of FGF-2 and FGF-2/HS treatments on FGFR-1-positive cells. The photographs were taken from the area adjacent to the wound cavity (arrows at the edge), which was filled with a piece of Gelfoam embedded with the experimental solution. $A$, Sample section from a control animal showing the distribution of FGFR-1 staining by the periphery of the injury site (arrows). FGFR-1 staining was light and sparse, mostly located in astrocytes. $B$, High magnification of $A$ displaying the typical morphology of FGFR-1-stained cells. $C$, Effects of recombinant FGF-2 treatment on the distribution and morphology of FGFR-1-immunostained cells. FGF-2 applied within the wound cavity caused an increase in number and intensity of FGFR-1 staining in astrocytes. $D$, High magnification of $C$ showing the effects of FGF-2 treatment on the phenotypic expression of FGFR-1-positive cells. There was an increase in density number of FGFR-1 cells, and their processes stained stronger as compared to treatments with saline or HS (not shown). E, Effect of the combined application of HS and FGF-2. There was an increase in the density number of FGFR-1-positive cells, and intensity of FGFR-1 staining on their processes as compared either with saline, HS, or FGF-2 treatments. $F$, High magnification of $E$ showing the morphology of FGFR-1-stained astrocytes. Scale bar (in $E$ ), $60 \mu \mathrm{m}$ for $A$, $C$, and $E ; 15 \mu \mathrm{m}$ for $B, D$, and $F$.

velopment (Herdon and Lander, 1990; Sheppard et al., 1991; Lander, 1993; Fuxe et al., 1994; Letourneau et al., 1994). Therefore, the interaction of the FGF system with the extracellular matrix and particular glycosoaminoglycans may rep- resent an efficient physiological mechanism to regulate the function of FGF-2 in specific cells.

There are factors other than HS in the cell environment that interact with FGF-2 and appear to modulate its action. In fact, 


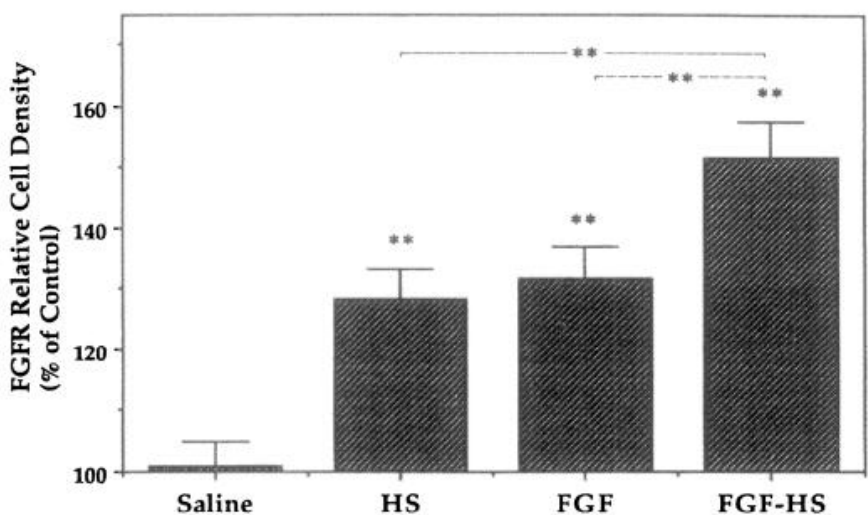

Figure 6. Quantification of the number of FGFR-1-immunostained cells by the area surrounding the wound cavity. The graph shows the effects of HS, FGF-2, and FGF/HS on the density of FGFR-1-positive astrocytic-like cells, expressed as a percentage of control (saline). FGF-2 treatment elicited an increase in the number of FGFR-1 cells compared with saline treatment. The application of HS also elicited an increase in FGFR-1 cell density as compared with saline controls. The effects of FGF-2 and HS were potentiated by the combined application of FGF/HS. Statistical comparisons were performed using ANOVA and Fisher's test $(* *, P<0.01)$. Error bars are SD.

emerging evidence suggests that the action of FGF-2 is associated with molecular cascades involving other growth factor molecules (Cross and Dexter, 1991). For example, the FGF family has other members such as FGF-1 and FGF-5 that interact with common receptors. It has been reported that FGF-1 and FGF-5 compete with FGF-2 for the binding to the FGFR-1 receptor (Clements et al., 1993), suggesting that a balance between different factors and FGFR-1 regulates their respective functions. There are also several described interactions between FGF-2 and other growth factor such as NGF, PDGF, TGF $\beta 1$, EGF, CNTF, and LIF that are associated with the physiology of astrocytes. For example, in contrast to FGF-2, application of TGF $\beta 1$ into a wound cavity dramatically decreases astrocytosis (Lindholm et al., 1992). Paradoxically, TGF $\beta 1$ probably indirectly helps FGF-2 by protecting the integrity of the extracellular matrix (Sporn and Roberts, 1991). The interaction between FGF-2 and TGF $\beta 1$ can be complex considering that TGF $\beta 1$ may potentiate (Labourdette et al., 1990; Lefebvre et al., 1991; Saunders and D'Amore, 1991) or inhibit (Baird and Durkin, 1986) the action of FGF-2 on cells. TGF $\beta 1$ can enhance the responsiveness to FGF-2 in certain cells, that is, astroblasts that are normally insensitive to FGF-2 (Labourdette et al., 1990). Therefore, the elucidation of the role of FGF-2 in molecular cascades is important to identify the signals that control astrocytosis and thus to design appropriate therapeutic applications.

Information presented in this study suggests a pivotal role of FGF-2 in events related to control of astrocytosis in vivo. According to studies in vitro the action of FGF-2 on astrocytes is diverse and includes effects on proliferation (Morrison et al., 1986), migration (Senior et al., 1986), and regulation of other trophic molecules (Yoshida and Gage, 1991). During development, the presence of FGF-2 has been shown to be a determining factor for the differentiation of progenitor cells into type- 2 astrocytes or oligodendrocytes (Lillien and Raff, 1990; McKinnon et al., 1990). According to our studies in vivo (Gómez-Pinilla et al., 1994), FGF-2 phenotypic expression appears on astrocytes by the time of proliferation, supporting a role of FGF-2 in this process. The present study suggests that FGF-2 and its receptor

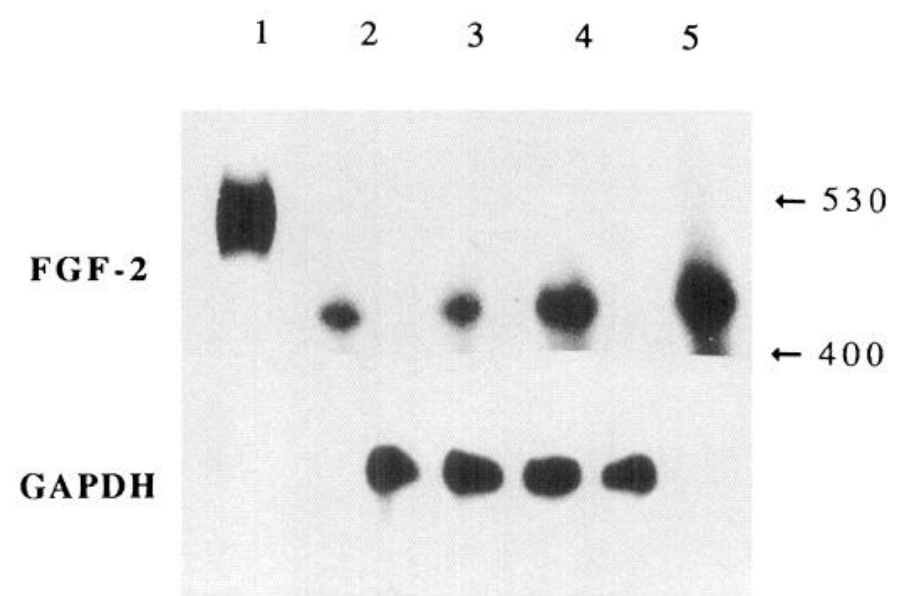

Figure 7. Comparisons of changes in FGF-2 mRNA elicited by HS, FGF-2, and FGF-2/HS treatments evaluated using nuclease protection assay. A wound cavity in the cerebral cortex was filled with a piece of Gelfoam embedded with saline (lane 2), HS (lane 3), FGF-2 (lane 4), or FGF/HS (lane 5), and the tissue surrounding the wound cavity was assayed with a nuclease protection assay. The treatment with FGF-2 triggered an increase in FGF-2 mRNA (lane 4), and this effect was potentiated by the combined application of FGF-2 and HS (lane 5). A 524 base ${ }^{32} \mathrm{P}$-labeled antisense probe that contained a 477 base FGF-2 gene coding region was used to assess relative levels of FGF-2 mRNA expression. Lane 1 of the gel displays the unprotected FGF-2 cRNA probe, and lanes 2-5 display the protected FGF-2 cRNA fragment. Size markers are indicated at the far right as a reference. At the bottom is shown a separate assay performed using the same total RNA hybridized with a GAPDH cRNA probe, to control for using equal amounts of RNA for each condition.

are involved in the process of astrocyte reactivity and/or proliferation in vivo, and this action appears to be regulated by HS. Therefore, FGF-2 via interaction with extracellular matrix components, and probably other factors, may be involved in a cascade of events that regulate development and plasticity of glial and neuronal cells in the CNS.

\section{References}

Anderson KJ, Dam D, Lee S, Cotman CW (1988) Basic fibroblast growth factor prevents death of lesioned cholinergic neurons in vivo. Nature 332:360-361.

Baird A, Durkin T (1986) Inhibition of endothelial cell proliferation by type- $\beta$ transforming growth factor: interactions with acidic and basic fibroblast growth factors. Biochem Biophys Res Commun 138: $476-482$

Chomczynski P, Sacchi N (1986) Single step method of RNA isolation by acid guanidinium thiocyanate-phenol-chloroform extraction. Anal Biochem 162:156-159.

Clements DA, Wang J-K, Dionne CA, Goldfarb M (1993) Activation of fibroblast growth factor (FGF) receptors by recombinant human FGF-5. Oncogene 8:1311-1316.

Cross M, Dexter T (1991) Growth factors in development, transformation, and tumorigenesis. Cell 64:271-280.

Dionne CA, Crumley G, Bellot F, Kaplow JM, Searfoss G, Ruta M, Burgess WH, Jaye M, Schlessinger J (1990) Cloning and expression of two distinct high-affinity receptors cross-reacting with acidic and basic fibroblast growth factors. EMBO J 9:2685-2692.

Flaumenhaft R, Moscatelli D, Rifkin DB (1990) Heparin and heparan sulfate increase the radius of diffusion and action of basic fibroblast growth factor. J Cell Biol 111:1651-1659.

Flott-Rahmel B, Gerdes W, Pechan PA, Brysch W, Schlingensiepen H, Seifert W (1992) BFGF induces its own gene expression in astrocytic and hippocampal cultures. Neuroreport 3:1077-1080.

Fuxe K, Chadi G, Tinner B, Agnati LF, Pettersson R, David G (1994) On the regional distribution of heparan sulfate proteoglycan immunoreactivity in the rat brain. Brain Res 636:131-138. 
Gerdes W, Brysch W, Schlingensiepen K-H, Seifert W (1992) Antisense bFGF oligodeoxynucleotides inhibit DNA synthesis of rat astrocytes. Neuroreport 3:43-46.

Gómez-Pinilla F, Cotman CW (1992) Transient lesion-induced increase of bFGF and its receptor in layer VIb (subplate cells) of the adult rat cerebral cortex. Neuroscience 49:771-780.

Gómez-Pinilla F, Won-Kyun Lee J, Cotman CW (1992) Basic FGF in adult rat brain: cellular distribution and response to entorhinal lesion and fimbria-fornix transection. J Neurosci 12:345-355.

Gómez-Pinilla F, Won-Kyun Lce J, Cotman CW (in press) Ccllular distribution of FGF-2 in the developing rat brain. Neuroscience 61: 911-923.

Gospadorowicz D, Cheng J (1986) Heparin protects basic and acidic FGF from inactivation. J Cell Physiol 128:475-484.

Grothe C, Otto D, Unsicker K (1989) Basic fibroblast growth factor promotes in vitro survival and cholinergic development of septal neurons: comparisons with the effects of nerve growth factor. Neuroscience 31:649-661.

Herndon ME, Lander AD (1990) A diverse set of proteoglycans are developmentally regulated in the rat central nervous system. Neuron $4: 949-961$

Hondermarck H, Deudon E, Boilly B (1992) Embryonic brain-derived heparan sulfate inhibits cellular membrane binding and biological activity of basic fibroblast growth factor. Dev Brain Res 68:247-253.

Hou J, Kan M, McKeehan K, McBride G, Adams P, McKeehan WL (1991) Fibroblast growth factor receptors from liver vary in three structural domains. Science 251:665-668.

Kan M, Wang F, Xu J, Crabb JW, Hou J, McKeehan WL (1993) An essential heparin-binding domain in the fibroblast growth factor receptor kinase. Science 259:1918-1921.

Keegan K, Johnson DE, Williams LT, Hayman MJ (1991) Isolation of an additional member of the fibroblast growth factor receptor family, FGFR-3. Proc Natl Acad Sci USA 88:1095-1099.

Klagsbrun M, Baird A (1991) A dual receptor system is required for basic fibroblast growth factor activity. Cell 67:229 231

Labourdette G, Janet T, Laeng P (1990) Transforming growth factor type $\beta 1$ modulates the effects of basic fibroblast growth factor on growth and phenotypic expression of rat astroblasts in vitro. J Cell Physiol 144:473-484.

Lander AD (1993) Proteoglycans in the nervous system. Curr Opin Neurobiol 3:716-723.

Lee PL, Johnson DE, Cousen LS, Fried VA, Williams LT (1989) Purification and complementary DNA cloning of a receptor for basic fibroblast growth factor. Science 245:57-60.

Lefebvre P, Van de Water T, Weber T (1991) Growth factor interactions in cultures of dissociated adult acoustic ganglia: neurotrophic effects. Brain Res 567:306-312.

Letourneau PC, Condic ML, Snow DM (1994) Interactions of developing neurons with the extracellular matrix. J Neurosci 14:915-928.

Lillien LE, Raff MC (1990) Differentiation signals in the CNS: type-2 astrocyte development in vitro as a model system. Neuron 5:111119.

Lindholm D, Castrén E, Kiefer R, Zafra F, Thoenen H (1992) Transforming growth factor- $\beta 1$ in the rat brain: increase after injury and inhibition of astrocyte proliferation. J Cell Biol 117:395-400.

Logan A, Frautschy SA, Gonzalez A-M, Baird A (1992) A time course for the focal elevation of synthesis of basic fibroblast growth factor and one of its high-affinity receptors $(\mathrm{fg})$ following a localized cortical brain injury. J Neurosci 12:3828-3837.

Mansukhani A, Dell'Era P, Moscatclli D, Kornbluth S, Hanafusa H, Basilico C (1992) Characterization of the murine BEK fibroblast growth factor (FGF) receptor: activation by three members of the FGF family and the requirement for heparin. Proc Natl Acad Sci USA 89:3305-3309.

Matsuzaki K, Yoshitake Y, Matuo Y, Sasaki H, Nishikawa K (1989) Monoclonal antibodies against heparin-binding growth factor II/basic fibroblast growth factor that block its biological activity: invalidity of the antibodies for tumor angiogenesis. Proc Natl Acad Sci USA 86:9911-9915.

McKinnon RD, Matsui T, Dubois-Dalcq M, Aaronson SA (1990) FGF modulates the PDGF-driven pathway of oligodendrocyte development. Neuron 5:603-614.

Morrison RS, De Vellis J (1981) Growth of purified astrocytes in a chemically defined medium. Proc Natl Acad Sci USA 78:7205-7209.

Moscatelli D (1987) High and low affinity binding sites for basic fibroblast growth factor on cultured cells: absence of a role for low affinity binding in the stimulation of plasminogen activator production by bovine capillary endothelial cells. J Cell Physiol 131:123130.

Nurcombe V, Ford MD, Wildschut JA, Barlett PF (1993) Developmental regulation of neural response to FGF-1 and FGF-2 by heparan sulfate proteoglycan. Science 260:103-106.

Otto D, Frotscher M, Unsicker K (1989) Basic fibroblast growth factor and nerve growth factor administered in Gelfoam rescue medial septal neurons after fimbria fornix transection. J Neurosei Res 22:8391.

Partanen J, Makela TP, Eerola E, Korhonen J, Hirvonen H, ClaessonWelsh L, Alitalo K (1991) FGFR-4, a novel acidic fibroblast growth factor receptor with a distinct expression pattern. EMBO J 10:13471354.

Rapracger AC, Krufka A, Olwin BB (1991) Requirement of heparan sulfate for bFGF-mediated fibroblast growth and myoblast differentiation. Science 252:1705-1708.

Ruoslahti E, Yamagushi Y (1991) Proteoglycans as modulators of growth factor activities. Cell 64:867-869.

Saunders KB, D'Amore PA (1991) FGF and TGF-beta: actions and interactions in biological systems. Crit Rev Eukaryotic Gene Expression 1:157-172.

Senior RM, Huang SS, Griffin GL, Huang JS (1986) Brain-derived growth factor is a chemoattractant for fibroblast and astroglial cells. Biochem Biophys Res Commun 141:67-72.

Sheppard AM, Hamilton SK, Pearlman AL (1991) Changes in the distribution of extracellular matrix components accompany early morphogenetic events of mammalian cortical development. J Neurosci 11:3928-3942.

Shimasaki S, Emoto M, Koba A, Mercado M, Shibata F, Cooksey K, Baird A, Ling N (1988) Complementary DNA cloning and sequencing of rat ovarian basic fibroblast growth factor and tissue distribution study of its mRNA. Biochem Biophys Res Commun 157:256-263.

Sievers J, Hausmann B, Unsicker K, Berry M (1987) Fibroblast growth factors promote the survival of adult rat retinal ganglion cells after transection of the optic nerve. Neurosci Lett 76:157-162.

Sporn MB, Roberts AB (1991) The transforming growth factor- $\beta s$. In: Peptide growth factors and their receptors I. (Sporn MB, Roberts AB, eds), pp 419-472. New York: Springer.

Ullrich A, Schlessinger J (1990) Signal transduction by receptors with tyrosine kinase activity. Cell 61:203-212.

Van der Wal EA, Gómez-Pinilla F, Cotman CW (1994) Seizure-associated induction of basic fibroblast growth factor and its receptor in the rat brain. Neuroscience 60:311-323.

Walicke PA (1988a) Interactions between basic fibroblast growth factor (FGF) and glycosoaminoglycans in promoting neurite outgrowth. Exp Neurol 102:144-148.

Walicke PA (1988b) Basic and acidic fibroblast growth factors have trophic effects on neurons from multiple CNS regions. J Neurosci 8:2618-2627.

Wanaka A, Johnson EM Jr, Milbrandt J (1990) Localization of FGF receptor mRNA in the adult rat central nervous system by in situ hybridization. Neuron 5:267-281.

Yayon A, Klagsbrun M, Esko JD, Leder P, Ornitz M (1991) Cell surface, heparin-like molecules are required for binding of basic fibroblast growth factor to its high affinity receptor. Cell 64:841-848.

Yoshida K, Gage F (1991) Fibroblast growth factors stimulate nerve growth factor synthesis and secretion by astrocytes. Brain Res 538: $118-126$. 